\title{
Notes on Cardinals That Are Characterizable by a Complete (Scott) Sentence
}

\author{
Ioannis Souldatos
}

\begin{abstract}
This is the first part of a study on cardinals that are characterizable by Scott sentences. Building on previous work of Hjorth, Malitz, and Baumgartner, we study which cardinals are characterizable by a Scott sentence $\varphi$, in the sense that $\varphi$ characterizes $\kappa$, if $\varphi$ has a model of size $\kappa$ but no models of size $\kappa^{+}$.

We show that the set of cardinals that are characterized by a Scott sentence is closed under successors, countable unions, and countable products (see Theorems 3.3 and 4.6 and Corollary 4.8). We also prove that if $\boldsymbol{\aleph}_{\alpha}$ is characterized by a Scott sentence, at least one of $\boldsymbol{\aleph}_{\alpha}, \boldsymbol{\aleph}_{\alpha+1}$, or $\left(\boldsymbol{\aleph}_{\alpha+1}, \boldsymbol{\aleph}_{\alpha}\right)$ is homogeneously characterizable (see Definitions 1.3 and 1.4 and Theorem 3.19). Based on an argument of Shelah, we give counterexamples that characterizable cardinals are not closed under predecessors or cofinalities.
\end{abstract}

\section{Introduction and Known Results}

This section contains the basic definitions and background theorems. A similar discussion also appears in Souldatos [9].

Let the signature of our logic be $\mathscr{L}$. We will consider only countable $\mathscr{L}$. For basic definitions in infinitary logic $\mathscr{L}_{\omega_{1}, \omega}$, the reader can refer to Keisler [4]. In $\mathscr{L}_{\omega_{1}, \omega}$ we allow formulas that have negation, universal/existential quantification, and countably long disjunctions and conjunctions but not countably long quantification.

Definition 1.1 A sentence $\sigma \in \mathscr{L}_{\omega_{1}, \omega}$ is called complete if for every sentence $\tau \in \mathscr{L}_{\omega_{1}, \omega}$, either $\sigma \Rightarrow \tau$ is valid or $\sigma \Rightarrow \neg \tau$ is valid.

Obviously, all complete sentences that hold inside the same model are equivalent to one another. Scott (in [7]) proved that for every countable model $\mathcal{M}$, there is a sentence $\varphi_{\mathcal{M}} \in \mathscr{L}_{\omega_{1}, \omega}$, such that if $\mathcal{N}$ is a countable model that also satisfies $\varphi_{\mathcal{M}}$,

Received May 4, 2012; accepted January 13, 2013

2010 Mathematics Subject Classification: Primary 03C75, 03C30; Secondary 03C35, 03E10, 03E75

Keywords: infinitary logic, Scott sentence, complete sentence, characterizable cardinals (C) 2014 by University of Notre Dame $\quad 10.1215 / 00294527-2798727$ 
then $\mathcal{M}$ and $\mathcal{N}$ are isomorphic; $\varphi_{\mathcal{M}}$ is called the Scott sentence for $\mathcal{M}$. From this it follows that every Scott sentence is a complete sentence.

Compactness fails in $\mathscr{L}_{\omega_{1}, \omega}$, and the same is true for the upward LöwenheimSkolem theorem. So, it can be the case that a certain sentence has models in cardinality $\boldsymbol{\aleph}_{\alpha}$ but not in cardinality $\boldsymbol{\aleph}_{\alpha+1}$. This motivates the following definition.

Definition 1.2 We say that an $\mathscr{L}_{\omega_{1}, \omega}$-sentence $\varphi$ characterizes $\boldsymbol{\aleph}_{\alpha}$, or that $\boldsymbol{\aleph}_{\alpha}$ is characterizable, if $\varphi$ has models in all cardinalities up to $\aleph_{\alpha}$ but not in cardinality $\boldsymbol{\aleph}_{\alpha+1}$. If $\varphi$ is the Scott sentence of a countable model (or any other complete sentence), we say that it completely characterizes $\boldsymbol{\aleph}_{\alpha}$ or that $\boldsymbol{\aleph}_{\alpha}$ is completely characterizable. Moreover, if $\varphi$ is the Scott sentence of a countable model $\mathcal{M}$, we also say that $\mathcal{M}$ characterizes $\aleph_{\alpha}$.

Denote by $\mathcal{C} \mathscr{H}_{\omega_{1}, \omega}$, the set of all completely characterizable cardinals.

Note that the downward Löwenheim-Skolem theorem still holds, which means that every sentence that characterizes some cardinal $\boldsymbol{\aleph}_{\alpha}$ has models in all cardinalities $\leq \boldsymbol{\aleph}_{\alpha}$.

On the other hand, Hanf [2] was the first to notice that there exists a cardinal, denote it $\mathscr{H}\left(\mathscr{L}_{\omega_{1}, \omega}\right)$, such that if an $\mathscr{L}_{\omega_{1}, \omega}$-sentence has a model of this cardinality, then it has models of all cardinalities. $\mathscr{H}\left(\mathscr{L}_{\omega_{1}, \omega}\right)$ is called the Hanf number for $\mathscr{L}_{\omega_{1}, \omega}$, and it is equal to $\beth_{\omega_{1}}$. So, $\mathcal{C} \mathscr{H}_{\omega_{1}, \omega} \subset \beth_{\omega_{1}}$, and from now on we only consider cardinals below $\beth_{\omega_{1}}$. We will also restrict ourselves to cardinals that are completely characterizable, and we may refer to them as just characterizable cardinals.

Using a stronger notion of characterizability which we will call homogenous characterizability, Malitz [6] and Baumgartner [1] proved that for all $\alpha<\omega_{1}, \beth_{\alpha+1}$ is homogeneously characterizable. We give the definition first.

Definition 1.3 If $P$ is a unary predicate symbol, we say that it is completely homogeneous for the $\mathscr{L}$-structure $\mathcal{A}$, if $P^{\mathcal{A}}=\{a \mid \mathcal{A} \models P(a)\}$ is infinite and every permutation of it extends to an automorphism of $\mathcal{A}$.

If $\kappa$ is a cardinal, we will say that $\kappa$ is homogeneously characterizable by $\left(\varphi_{\kappa}, P_{\kappa}\right)$, if $\varphi_{\kappa}$ is a complete $\mathscr{L}_{\omega_{1}, \omega}$-sentence and $P_{\kappa}$ a unary predicate in the language of $\varphi_{\kappa}$ such that

- $\varphi_{\kappa}$ does not have models of power greater than $\kappa$;

- if $\mathcal{M}$ is the (unique) countable model of $\varphi_{\kappa}$, then $P_{\kappa}$ is infinite and completely homogeneous for $\mathcal{M}$; and

- there is a model $\mathcal{A}$ of $\varphi_{\kappa}$ such that $P_{\kappa}^{\mathcal{A}}$ has cardinality $\kappa$.

Denote the set of all homogeneously characterizable cardinals by $\mathscr{H}_{\mathcal{C}} \mathscr{H}_{\omega_{1}, \omega}$.

We will also need a refinement of the above definition.

Definition 1.4 If $\kappa \geq \lambda$ are infinite cardinals, we will say that $(\kappa, \lambda)$ is homogeneously characterizable by $\left(\varphi_{\kappa}, P_{\lambda}\right)$, if $\varphi_{\kappa}$ is a complete $\mathscr{L}_{\omega_{1}, \omega}$-sentence and $P_{\lambda}$ a unary predicate in the language of $\varphi_{\kappa}$ such that

- $\varphi_{\kappa}$ does not have models of power greater than $\kappa$;

- in all models of $\varphi_{\kappa}$ the predicate $P_{\lambda}$ has size at most $\lambda$;

- if $\mathcal{M}$ is the (unique) countable model of $\varphi_{\kappa}$, then $P_{\lambda}$ is infinite and completely homogeneous for $\mathcal{M}$;

- there is a model $\mathcal{A}$ of $\varphi_{\kappa}$ of size $\kappa$; and

- there is a model $\mathscr{B}$ of $\varphi_{\kappa}$ such that $P_{\lambda}^{\mathcal{B}}$ has cardinality $\lambda$. 
Observe that we do not require that $\mathcal{A}$ and $\mathcal{B}$ are the same model; that is, the maximum cardinality for $\varphi_{\kappa}$ and $P_{\lambda}$ may be attained at different models.

Abusing notation we will write $(\kappa, \lambda) \in \mathscr{H}^{\mathcal{C}} \mathscr{H}_{\omega_{1}, \omega}$ for the above, and $(\kappa, \kappa) \in$ $\mathscr{H} \mathcal{H} \mathscr{H}_{\omega_{1}, \omega}$ is equivalent to $\kappa \in \mathscr{H}^{C} \mathscr{H}_{\omega_{1}, \omega}$.

Obviously, $\mathscr{H}^{\mathcal{C}} \mathscr{H}_{\omega_{1}, \omega} \subset \mathcal{C} \mathscr{H}_{\omega_{1}, \omega}$, but the inverse inclusion fails, with $\aleph_{0}$ being a counterexample (see Hjorth [3]). It is open whether there is any other counterexample. By Corollary 3.21 it is consistent that all such counterexamples have cofinality $\omega$. Our conjecture is that a characterizable cardinal is not homogeneously characterizable if and only if it has cofinality $\omega$ (see Conjecture 1).

In [6], Malitz proved that under the assumption of the generalized continuum hypothesis $(\mathrm{GCH})$, for every successor $\alpha<\omega_{1}, \beth_{\alpha}$ is homogeneously characterizable. Baumgartner improved this result in [1] by eliminating the $\mathrm{GCH}$ assumption.

Hjorth in [3] extended a result of Knight [5] that $\aleph_{1}$ is characterizable, to all $\aleph_{\alpha}$ 's being characterizable, for $\alpha$ countable.

Breaking the arguments down we can easily get the following generalizations. Baumgartner's argument proves that the class of homogeneously characterizable cardinals is closed under the powerset operator; that is, if $\aleph_{\alpha} \in \mathscr{H}^{\mathcal{C}} \mathscr{H}_{\omega_{1}, \omega}$, then $2^{\boldsymbol{\aleph}_{\alpha}} \in \mathscr{H}^{\mathcal{C}} \mathscr{H}_{\omega_{1}, \omega}$ (see Theorem 4.1). Hjorth's argument proves that the class of characterizable cardinals is closed under successors and countable unions; that is, if $\aleph_{\alpha} \in \mathcal{C} \mathscr{H}_{\omega_{1}, \omega}$ and $\beta<\omega_{1}$, then $\boldsymbol{\aleph}_{\alpha+\beta} \in \mathcal{C} \mathscr{H}_{\omega_{1}, \omega}$ (see Theorem 3.3). This means that characterizable cardinals come in clusters of length $\omega_{1}$.

Definition 1.5 A cardinal $\aleph_{\alpha} \in \mathcal{C} \mathscr{H}_{\omega_{1}, \omega}$ is called the head of a cluster if we cannot find ordinals $\beta, \gamma$ such that

- $\boldsymbol{\aleph}_{\gamma} \in \mathcal{C} \mathscr{H}_{\omega_{1}, \omega}$,

- $\beta<\omega_{1}$, and

- $\aleph_{\alpha}=\aleph_{\gamma+\beta}$.

It is immediate that all characterizable cardinals are of the form $\boldsymbol{\aleph}_{\alpha+\beta}$, where $\boldsymbol{\aleph}_{\alpha}$ is the head of a cluster and $\beta<\omega_{1}$.

Since not all characterizable cardinals are homogeneously characterizable, the theorems by Hjorth and Baumgartner cannot be combined directly.

Our contributions: Malitz's proof that $2^{\aleph_{0}}$ is (homogeneously) characterizable generalizes to the following: if $\lambda$ is characterizable, then $\lambda^{\omega}$ is homogeneously characterizable. Using this theorem, we prove closure under countable products for both the characterizable and homogeneously characterizable cardinals (see Corollary 4.8). Moreover, if $\boldsymbol{\aleph}_{\alpha}^{\boldsymbol{\aleph}_{\beta}} \in \mathcal{C} \mathscr{H}_{\omega_{1}, \omega}$, then for all $\gamma<\omega_{1}, \boldsymbol{\aleph}_{\alpha+\gamma}^{\boldsymbol{\aleph}_{\beta}} \in \mathcal{H}^{\mathcal{C}} \mathscr{H}_{\omega_{1}, \omega}$ (see Theorem 4.9). In particular if $\aleph_{\alpha}$ is the head of a cluster and $\boldsymbol{\aleph}_{\alpha}^{\aleph_{\beta}}$ is a cardinal in $\mathcal{C} \mathscr{H}_{\omega_{1}, \omega}$, then the same is true if we replace $\boldsymbol{\aleph}_{\alpha}$ with any cardinal in the same cluster. We then conclude that if $\ell$ is the smallest set of characterizable cardinals that contains $\aleph_{0}$ and is closed under successors, countable unions, countable products, and powersets, then it is also closed under powers. This is Theorem 4.10. Whence, we see that the class of characterizable and homogeneously characterizable cardinals may be much richer than just containing the countable aleph and beth numbers. Of course, it depends on our set-theoretic universe.

In the last section we prove that characterizable cardinals are not closed under predecessors and cofinalities. 


\section{Structure of the Paper}

- In Section 3, most of the theorems either follow, or extend, theorems from [3]. The main theorem is that for every $\kappa \in \mathcal{C} \mathscr{H}_{\omega_{1}, \omega}$, at least one of $\kappa, \kappa^{+}$or $\left(\kappa^{+}, \kappa\right)$ is in $\mathscr{H}^{\mathcal{C}} \mathscr{H}_{\omega_{1}, \omega}$. We will use some of the theorems from this section in Part II too.

- In Section 4 the main theorem is that for $\kappa \in \mathcal{C} \mathscr{H}_{\omega_{1}, \omega}, \kappa^{\omega}$ is in $\mathscr{H} \mathcal{C} \mathscr{H}_{\omega_{1}, \omega}$. The construction behind it is given in Theorem 4.6 and is very similar to the construction found in [6]. Some consequences of this theorem are proved too.

- Section 5 contains counterexamples that characterizable cardinals are not closed under predecessors or cofinalities.

- Section 6 contains a few open questions about $(\mathcal{M}, \mathcal{N})$-full structures formulated as questions about functions.

\section{Successors}

In this section we deal with successors of characterizable cardinals. The main theorem is Theorem 3.19: If $\kappa \in \mathcal{C} \mathscr{H}_{\omega_{1}, \omega}$, then at least one of the following holds: $\kappa \in \mathscr{H}^{\mathcal{C}} \mathscr{H}_{\omega_{1}, \omega}, \kappa^{+} \in \mathscr{H} \mathcal{H} \mathscr{H}_{\omega_{1}, \omega}$, or $\left(\kappa^{+}, \kappa\right) \in \mathscr{H} \mathcal{H} \mathscr{H}_{\omega_{1}, \omega}$.

The first two theorems are (essentially) in [3], although Hjorth is interested only in the case where $\alpha<\omega_{1}$.

Theorem 3.1 (Hjorth) $\quad$ If $\aleph_{\alpha} \in \mathcal{C} \mathscr{H}_{\omega_{1}, \omega}$, then $\aleph_{\alpha+1} \in \mathcal{C} \mathscr{H}_{\omega_{1}, \omega}$.

Proof This follows by (the proof of ) [3, Theorem 5.1].

Theorem 3.2 (Hjorth) Whenever $\boldsymbol{\aleph}_{\alpha_{n}}, n \in \omega$, is a nondecreasing sequence of cardinals in $\mathcal{C} \mathscr{H}_{\omega_{1}, \omega}$, then $\aleph_{\lambda}=\sup \aleph_{\alpha_{n}}$ is also in $\mathcal{C} \mathscr{H}_{\omega_{1}, \omega}$.

Proof Take the disjoint union of structures that characterize $\aleph_{\alpha_{n}}$, for all $n$.

Combining these two theorems we get the following by induction on $\beta$.

Theorem 3.3 If $\aleph_{\alpha} \in \mathcal{C} \mathscr{H}_{\omega_{1}, \omega}$, then $\aleph_{\alpha+\beta} \in \mathcal{C} \mathscr{H}_{\omega_{1}, \omega}$, for $\beta<\omega_{1}$.

So, characterizable cardinals come into clusters of length $\omega_{1}$.

Next, for the sake of completeness, we repeat some definitions from [3], as well as a few lemmas.

Definition 3.4 Let $\mathcal{M}, \mathcal{N}$ be structures with languages $\mathscr{L}_{\mathcal{M}}, \mathscr{L}_{\mathcal{N}}$, respectively. Assume that $\mathscr{L}_{\mathcal{M}}$ and $\mathscr{L}_{\mathcal{N}}$ have no common symbols and are entirely relational. Let $S$ be a ternary relation, let $P$ be a binary relation, and for every $k \in \omega$, let $T_{k}$ be a $(k+2)$-ary relation, all of which are new symbols and do not appear in $\mathscr{L}_{\mathcal{M}}$ or $\mathscr{L}_{\mathcal{N}}$. Let $\mathscr{L}\left(\mathscr{L}_{\mathcal{M}}, \mathscr{L}_{\mathcal{N}}\right)$ be the language generated by $\mathscr{L}_{\mathcal{M}}, \mathscr{L}_{\mathcal{N}}, S, P,\left\{T_{k}: k \in \omega\right\}$. Let an $\mathscr{L}\left(\mathscr{L}_{\mathcal{M}}, \mathscr{L}_{\mathcal{N}}\right)$-structure $\mathcal{A}$ including $\mathcal{M}, \mathcal{N}$ be $(\mathcal{M}, \mathcal{N})$-neat if

(1) $\mathcal{A} \backslash(\mathcal{M} \cup \mathcal{N})$ is finite;

(2) for any relation symbol $R$ in $\mathscr{L}_{\mathcal{M}}$ and $\mathbf{a} \in \mathcal{A}$, if $\mathcal{A} \models R(\mathbf{a})$, then $\mathbf{a} \in \mathcal{M}$;

(3) similarly for any relation symbol $R$ in $\mathscr{L}_{\mathcal{N}}$ and $\mathbf{a} \in \mathcal{A}$, if $\mathcal{A} \models R(\mathbf{a})$, then $\mathbf{a} \in \mathcal{N} ;$

(4) if $\mathcal{A} \models T_{k}\left(a_{0}, a_{1}, \mathbf{b}\right)$, then $a_{0}, a_{1}, \mathbf{b} \in \mathcal{A} \backslash(\mathcal{M} \cup \mathcal{N})$;

(5) if $\mathcal{A} \models P(a, c)$, then $a \in \mathcal{N}$ and $c \notin(\mathcal{M} \cup \mathcal{N})$;

(6) if $\mathcal{A} \models S\left(a_{0}, c_{0}, c_{1}\right)$, then $a \in \mathcal{M}$ and $c_{0}, c_{1} \notin(\mathcal{M} \cup \mathcal{N})$; 
$\left(* 1^{\mathcal{M}, \mathcal{N}}\right) \mathcal{A}$ satisfies the conjunction of

$\left(* 1_{a}^{\mathcal{M}, \mathcal{N}}\right) \forall c_{0}, c_{1} \notin(\mathcal{M} \cup \mathcal{N}) \exists a \in \mathcal{M} S\left(a, c_{0}, c_{1}\right)$,

$\left(* 1_{b}^{\mathcal{M}, \mathcal{N}}\right) \forall c_{0}, c_{1} \forall a_{0} \neq a_{1} S\left(a_{0}, c_{0}, c_{1}\right) \Rightarrow \neg S\left(a_{1}, c_{0}, c_{1}\right)$;

$\left(* 2^{\mathcal{M}, \mathcal{N}}\right) \mathcal{A}$ satisfies the conjunction of

$\left(* 2_{a}^{\mathcal{M}, \mathcal{N}}\right)$ for each $k$ and $i<j<k$,

$\forall c_{0}, c_{1}, b_{0}, \ldots, b_{k-1} T_{k}\left(c_{0}, c_{1}, b_{0}, \ldots, b_{k-1}\right) \Rightarrow$

$\left(b_{i} \neq b_{j} \wedge c_{0} \neq c_{1}\right)$;

$\left(* 2_{b}^{\mathcal{M}, \mathcal{N}}\right)$ for each $k$ and permutation $\pi$ of $k$,

$\forall c_{0}, c_{1}, b_{0}, \ldots, b_{k-1} T_{k}\left(c_{0}, c_{1}, b_{0}, \ldots, b_{k-1}\right) \Leftrightarrow$

$T_{k}\left(c_{1}, c_{0}, b_{\pi(0)}, \ldots, b_{\pi(k-1)}\right)$,

$\left(* 2_{c}^{\mathcal{M}, \mathcal{N}}\right) \forall c_{0}, c_{1}, b_{0}, \ldots, b_{k-1} \forall a \in \mathcal{M} \bigwedge_{i<k} T_{k}\left(c_{0}, c_{1}, b_{0}, \ldots, b_{k-1}\right) \Rightarrow$ $\left(S\left(a, c_{0}, b_{i}\right) \Leftrightarrow S\left(a, c_{1}, b_{i}\right)\right)$,

$\left(* 2_{d}^{\mathcal{M}, \mathcal{N}}\right) \forall c_{0}, c_{1}, b_{0}, \ldots, b_{k-1} \forall d \forall a \quad \in \quad \mathcal{M} \quad\left(T_{k}\left(c_{0}, c_{1}, b_{0}, \ldots, b_{k-1}\right)\right.$ $\left.\bigwedge_{i<k} d \neq b_{i}\right) \Rightarrow\left(S\left(a, c_{0}, d\right) \Rightarrow \neg S\left(a, c_{1}, d\right)\right) ;$

$\left(* 3^{\mathcal{M}, \mathcal{N}}\right) \mathcal{A}$ satisfies the conjunction of

$\left(* 3_{a}^{\mathcal{M}, \mathcal{N}}\right) \forall c \notin(\mathcal{M} \cup \mathcal{N}) \exists a \in \mathcal{N} P(a, c)$,

$\left(* 3_{b}^{\mathcal{M}, \mathcal{N}}\right) \forall c \forall a_{0} \neq a_{1} \neg\left(P\left(a_{0}, c\right) \wedge P\left(a_{1}, c\right)\right)$;

(7) for all $c_{0}, c_{1} \in \mathcal{A} \backslash(\mathcal{M} \cup \mathcal{N})$ there is $k \in \omega$ and $\mathbf{b} \in \mathcal{A}$ with

$$
\mathcal{A} \models T_{k}\left(c_{0}, c_{1}, \mathbf{b}\right) \text {. }
$$

Definition 3.5 An $\mathscr{L}\left(\mathscr{L}_{\mathcal{M}}, \mathscr{L}_{\mathcal{N}}\right)$-structure $\mathcal{A}$ including $\mathcal{M}, \mathcal{N}$ is $(\mathcal{M}, \mathcal{N})$-happy if for all finite $A \subset \mathcal{A}$ there is some $(\mathcal{M}, \mathcal{N})$-neat substructure $\mathscr{B}$ of $\mathcal{A}$ with $\mathscr{B} \supset A$.

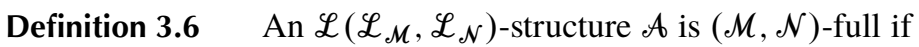

(1) it is $(\mathcal{M}, \mathcal{N})$-happy ;

(2) for all $(\mathcal{M}, \mathcal{N})$-neat $F \subset \mathcal{A}$ and $(\mathcal{M}, \mathcal{N})$-neat $H_{0} \supset F$ there is $(\mathcal{M}, \mathcal{N})$-neat $H_{1} \subset \mathcal{A}$ with $F \subset H_{1}$ and there is $i: H_{0} \cong H_{1}$ with $\left.i\right|_{F}$ the identity.

Observation 3.7 It follows from the definition that

(a) if $\mathcal{A}$ is $(\mathcal{M}, \mathcal{N})$-full, then

$$
\forall a \in \mathcal{N} \exists c \notin(\mathcal{M} \cup \mathcal{N}) P(a, c) ;
$$

in particular, $\mathcal{N}$ gives a partition of $\mathcal{A} \backslash(\mathcal{M} \cup \mathcal{N})$;

(b) combining $(a)$ and $\left(* 3^{\mathcal{M}, \mathcal{N}}\right),|\mathcal{N}| \leq|\mathcal{A} \backslash(\mathcal{M} \cup \mathcal{N})|$;

(c) if $\mathcal{A}$ is $(\mathcal{M}, \mathcal{N})$-full, $c \in A \backslash(\mathcal{M} \cup \mathcal{N})$, and $a \in \mathcal{M}$, then there exists some $c^{\prime} \in A \backslash(\mathcal{M} \cup \mathcal{N})$ such that $S\left(a, c, c^{\prime}\right)$; in particular, $|M| \leq|A \backslash(\mathcal{M} \cup \mathcal{N})|$ and combining with $(b),|\mathcal{M} \cup \mathcal{N}| \leq|A \backslash(\mathcal{M} \cup \mathcal{N})|$;

(d) fullness is expressible in $\mathscr{L}_{\omega_{1}, \omega}$; that is, there exists some $\mathscr{L}_{\omega_{1}, \omega}$-sentence $\chi$ such that $\mathcal{A}$ is a $(\mathcal{M}, \mathcal{N})$-full model, for some $\mathcal{M}, \mathcal{N}$ if and only if $A \models \chi$.

The following lemmas are [3, Corollary 5.2, Lemma 5.4, Lemma 5.5].

Lemma 3.8 (Hjorth) Let $\gamma \leq \kappa$ be cardinals, and let $\kappa$ be an infinite cardinal. If $\mathcal{M}$ is a structure of size $\kappa$ and $\mathcal{N}$ is a structure of size $\gamma$, then there exists an $(\mathcal{M}, \mathcal{N})$-full structure of cardinality $\kappa$.

Lemma 3.9 (Hjorth) Under the same assumptions for $\mathcal{M}$ and $\mathcal{N}$, there is no $(\mathcal{M}, \mathcal{N})$-full structure of size greater than $\kappa^{+}$. 
Lemma 3.10 (Hjorth) Let $|\mathcal{M}|=\boldsymbol{\aleph}_{0},|\mathcal{N}| \leq \boldsymbol{\aleph}_{0}$, and let $\mathcal{A}, \mathcal{B}$ be $(\mathcal{M}, \mathcal{N})$-full structures of size $\aleph_{0}$. Then $\mathcal{A} \cong \mathscr{B}$ and in fact there is an isomorphism $i: \mathcal{A} \cong \mathscr{B}$ with $\left.i\right|_{\mathcal{M}}$ and $\left.i\right|_{\mathcal{N}}$ both equal to the identity.

By inspecting the proof of Lemma 3.9, we see that the bound on $(\mathcal{M}, \mathcal{N})$-full structures is induced by the size of $\mathcal{M}$ and that $\mathcal{N}$ is not playing any role. This is not surprising since by Observation 3.7(a), $\mathcal{N}$ gives a partition of the elements in $\mathcal{A} \backslash(\mathcal{M} \cup \mathcal{N})$. So, we rephrase Lemma 3.9 as follows.

Lemma 3.11 Let $\kappa$ be an infinite cardinal, and let $\mathcal{M}$ be a structure of size $\kappa$. If $\mathcal{A}$ is $(\mathcal{M}, \mathcal{N})$-full for some $\mathcal{N}$, then $|\mathcal{A} \backslash(\mathcal{M} \cup \mathcal{N})| \leq \kappa^{+}$.

Corollary 3.12 The assumption on $\mathcal{N}$ in Lemma 3.9 can be relaxed to $|\mathcal{N}| \leq \kappa^{+}$. In particular, for any $\mathcal{N}$, all $(\mathcal{M}, \mathcal{N})$-full structures (if any) have size at most $\kappa^{+}$.

Working similarly to [3, Lemma 3.6], we can also prove the following.

Lemma $3.13 \quad$ Let $\mathcal{M}, \mathcal{N}$ be models of size $\aleph_{0}$, let $\mathcal{A}$ be the (unique) $(\mathcal{M}, \mathcal{N})$-full countable structure, and assume that $\mathscr{L}_{\mathcal{N}}=\emptyset$, that is, $\mathcal{N}$ comes with no structure on it. Then for any permutation $\pi: \mathcal{N} \rightarrow \mathcal{N}$, there exists an automorphism $\sigma: \mathcal{A} \rightarrow$ A such that

$$
\mathcal{A} \vDash P(a, c) \Leftrightarrow P(\pi(a), \sigma(c)) .
$$

In particular, $\mathcal{N}$ is completely homogeneous for $\mathcal{A}$ (see Definition 1.3).

Proof Let $\mathcal{B}$ be a countable model that agrees with $\mathcal{A}$ on all relations except for $P$; it holds that

$$
\mathscr{B} \models P(a, c) \quad \text { if and only if } \quad \mathcal{A} \models P(\pi(a), c) .
$$

It follows by the definition that $\mathscr{B}$ is also $(\mathcal{M}, \mathcal{N})$-full, and using Lemma 3.10, we find the required $\sigma$.

Definition 3.14 Let $\lambda$ be a cardinal number (finite or infinite). An $(\mathcal{M}, \lambda)$-neat structure is an $(\mathcal{M}, \mathcal{N})$-neat structure, where $\mathcal{N}$ has size $\lambda$ and $\mathscr{L}_{\mathcal{N}}=\emptyset$; that is, there is no structure on $\mathcal{N}$ other than the predicate $P$ given by Definition 3.4. Since the choice of $\mathcal{N}$ is immaterial, we will assume for the rest of the paper that the universe of $\mathcal{N}$ is $\lambda$.

Similarly define $(\mathcal{M}, \lambda)$-happy and $(\mathcal{M}, \lambda)$-full structures.

The following is immediate from the definition.

Lemma 3.15 Let $\kappa, \lambda$ be infinite cardinals.

(a) If there exists an $(\mathcal{M}, \mathcal{N})$-full structure of size $\kappa$ and $|\mathcal{N}|=\lambda$, then there exists an $(\mathcal{M}, \lambda)$-full structure of size $\kappa$.

(b) If there exists an $(\mathcal{M}, \lambda)$-full structure of size $\kappa$, then there exists an $(\mathcal{M}, \mu)$-full structure of size $\kappa$, for all $\mu \leq \lambda$.

If $|\mathcal{M}|=\kappa$, it is an open question whether for $\lambda<\kappa^{+}$the existence of some $(\mathcal{M}, \lambda)$-full structure of size $\kappa^{+}$implies the existence of some $\left(\mathcal{M}, \lambda^{\prime}\right)$-full structure of size $\kappa^{+}$where $\lambda^{\prime}>\lambda$, or even the existence of some $\left(\mathcal{M}, \kappa^{+}\right)$-full structure (necessarily of size $\kappa^{+}$). Theorem 3.18 gives some necessary conditions for this to be true. ${ }^{1}$ 
Definition 3.16 Let $K$ be a collection of structures, and let $\kappa$ be an infinite cardinal. $K$ has the $\left(<\aleph_{0},<\aleph_{0},<\kappa\right)$-disjoint amalgamation property (dap for short), if for every $A, B, C \in K$ such that $|A|<\aleph_{0},|B|<\aleph_{0},|C|<\kappa, A \subset B, C$, and $C \cap B=A$, there exists some $D \in K$ such that $D \supset C$ and there exists an embedding $i: B \rightarrow D$ such that $\left.i\right|_{A}=i d$ and $i[B] \cap C=A$.

Theorem 3.17 Let $\mathcal{A}$ be an $(\mathcal{M}, \mathcal{N})$-full structure, and let $K$ be the collection of all $(\mathcal{M}, \lambda)$-happy substructures of $\mathcal{A}$. The following are equivalent.

(I) $K$ satisfies the $\left(<\boldsymbol{\aleph}_{0},<\aleph_{0},<\lambda\right)$-dap.

(II) For all $A \subset \mathcal{A}$ and $B \supset A, A, B(\mathcal{M}, \mathcal{N})$-neat, there exist (distinct) substructures $C_{\alpha} \subset \mathcal{A}, \alpha<\lambda$ and isomorphisms $i_{\alpha}: B \cong C_{\alpha}$ such that $C_{\alpha} \cap C_{\beta}=A=C_{\alpha} \cap B$ for all $\alpha \neq \beta$ and $\left.i_{\alpha}\right|_{A}=i d$.

Proof $\quad(I) \Rightarrow(I I)$ Fix some $(\mathcal{M}, \mathcal{N})$-neat structures $A, B$ as in $(I I)$, and we will define the $C_{\alpha}$ 's by induction. Assume that for some $\beta<\lambda,\left(C_{\alpha}\right)_{\alpha<\beta}$ have been defined. We need to define $C_{\beta}$ too. Consider $C=\bigcup_{\alpha<\beta} C_{\alpha}$, and apply the $\left(<\aleph_{0},<\aleph_{0},<\lambda\right)$-dap for the triple $(A, B, C)$ to find some $(\mathcal{M}, \mathcal{N})$-happy $D \subset \mathcal{A}$ such that $D \supset C$ and there exists an embedding $i: B \rightarrow D$ such that $\left.i\right|_{A}=i d$ and $i[B] \cap C=A$. Let $C_{\beta}=i[B]$, and the result follows.

$(I I) \Rightarrow(I)$ Let $A, B, C$ be structures in $K$ such that $|A|<\aleph_{0},|B|<\aleph_{0}$, $|C|<\lambda, A \subset B, C$, and $C \cap B=A$. Applying (II) for $A, B$ we can find distinct substructures $C_{\alpha} \subset \mathcal{A}, \alpha<\lambda$ and isomorphisms $i_{\alpha}: B \cong C_{\alpha}$ such that $C_{\alpha} \cap C_{\beta}=A=C_{\alpha} \cap B$ for all $\alpha \neq \beta$ and $\left.i_{\alpha}\right|_{A}=i d$. Since $|C|<\lambda$ and there are $\lambda$-many such $C_{\alpha}$ 's, there exists some $\alpha<\lambda$ such that $C \cap C_{\alpha}=A$. Taking $D$ to be an $(\mathcal{M}, \mathcal{N})$-happy structure that contains $C \cup C_{\alpha}$ we finish the proof.

Abusing terminology we will refer to part (II) of Theorem 2.17 as: "for every pair $(A, B)$ of $(\mathcal{M}, \mathcal{N})$-neat structures there exist $\lambda$-many disjoint copies of $B$ in $\mathcal{A}$," even though the $C_{\alpha}$ 's in the previous theorem share $A$ as their common intersection.

Theorem 3.18 Let $\mathcal{M}$ be a model of size $\kappa$, and let $\lambda \leq \kappa^{+}$.

(a) If there exists an $(\mathcal{M}, \lambda)$-full structure of size $\kappa^{+}$, then there exists an $(\mathcal{M}, 1)$-full structure of size $\kappa^{+}$such that the collection $K$ of all its $(\mathcal{M}, 1)$-happy substructures satisfies the $\left(<\aleph_{0},<\aleph_{0},<\lambda\right)$-dap.

(b) If there exists an $(\mathcal{M}, 1)$-full structure of size $\kappa^{+}$such that the collection $K$ of all its $(\mathcal{M}, 1)$-happy substructures satisfies the $\left(<\boldsymbol{\aleph}_{0},<\boldsymbol{\aleph}_{0},<\kappa^{+}\right)$-dap, then there exists an $\left(\mathcal{M}, \kappa^{+}\right)$-full structure of size $\kappa^{+}$.

Proof (a) Let $\mathcal{A}$ be an $(\mathcal{M}, \lambda)$-full structure of size $\kappa^{+}$. By Observation 3.7, $\lambda$ gives a partition of $\mathcal{A} \backslash(\mathcal{M} \cup \mathcal{N})$, and we can consider $\mathcal{A}$ as an $(\mathcal{M}, 1)$-full structure too.

A word of caution: For the rest of the proof we will switch between considering $\mathcal{A}$ (and its substructures) as $(\mathcal{M}, \lambda)$-full and $(\mathcal{M}, 1)$-full. Abusing notation we will write $\mathcal{A}$ for both the $(\mathcal{M}, \lambda)$-full and the $(\mathcal{M}, 1)$-full structure, even though $(\mathcal{A} \backslash \lambda) \cup\{1\}$ is more appropriate for the latter. Other than that, it should be obvious from the context what we mean.

We have to prove that the collection $K$ of all $(\mathcal{M}, 1)$-happy substructures of $\mathcal{A}$ satisfies the $\left(<\aleph_{0},<\aleph_{0},<\lambda\right)$-dap. Let $A, B, C \in K$ be such that $|A|<\aleph_{0}$, $|B|<\aleph_{0},|C|<\lambda, A \subset B, C$, and $C \cap B=A$. Notice here that since for finite 
structures the notions of $(\mathcal{M}, \lambda)$-happy and $(\mathcal{M}, \lambda)$-neat coincide, $A, B$ are (both) $(\mathcal{M}, \lambda)$-neat and $(\mathcal{M}, 1)$-neat. Let $X$ be the set

$$
\{\alpha \in \lambda \mid \exists x \in C P(\alpha, x)\},
$$

where $C$ is seen here as an $(\mathcal{M}, \lambda)$-happy structure. It follows from Observation 3.7 that $|X| \leq|C|<\lambda$, and let $\beta$ be an element in $\lambda \backslash X$. Define a new structure $B^{\prime}$ that agrees with $B$ except for $P$, and let for all $x \in B^{\prime} \backslash A$,

$$
B^{\prime} \models P(\beta, x) .
$$

$B^{\prime}$ is also an $(\mathcal{M}, \lambda)$-neat structure, and it extends $A$. By the definition of $(\mathcal{M}, \lambda)$-fullness, there exists some $(\mathcal{M}, \lambda)$-neat $H \subset \mathcal{A}$ with $A \subset H$ and there exists some $i: B^{\prime} \cong H$ with $\left.i\right|_{A}$ being the identity. It follows that for all $x \in H \backslash A$, $P(\beta, x)$ and by the definition of $\beta$, for all $y \in C \backslash A, \neg P(\beta, y)$. Therefore, $C \cap H=A$. Moreover, if $B, B^{\prime}, H$ are seen as $(\mathcal{M}, 1)$-neat structures, $i$ becomes an isomorphism between $B$ and $H$ and $\left.i\right|_{A}=i d$. Letting $D=C \cup H$, where $D$ is seen as an $(\mathcal{M}, 1)$-structure too, we conclude part (a).

(b) Let $\mathcal{A}$ be an $(\mathcal{M}, 1)$-full structure of size $\kappa^{+}$, let $K$ be the collection of all ( $\mathcal{M}, 1)$-happy substructures of $\mathcal{A}$, and assume that $K$ satisfies the $\left(<\boldsymbol{\aleph}_{0},<\boldsymbol{\aleph}_{0}\right.$, $<\kappa^{+}$)-dap. By Theorem 3.17 (see also the comments after the theorem) for every pair $(A, B)$ of $(\mathcal{M}, 1)$-neat structures, there exist $\kappa^{+}$-many disjoint copies of $B$ in $\mathcal{A}$.

We can decompose $\mathcal{A}$ into $\left(\mathcal{A}_{\alpha}\right)_{\alpha<\kappa^{+}}$such that for every $\alpha<\kappa^{+}$,

(1) $\left|\mathcal{A}_{\alpha}\right|<\kappa^{+}$and $\alpha<\beta \rightarrow \mathcal{A}_{\alpha} \subset \mathcal{A}_{\beta}$;

(2) $\mathcal{A}=\bigcup_{\alpha} \mathcal{A}_{\alpha}$;

(3) for $\alpha$ limit, $\mathscr{A}_{\alpha}=\bigcup_{\alpha<\beta} \mathcal{A}_{\beta}$;

(4) for $\alpha$ successor, fix some $A \subset \mathcal{A}_{\alpha-1}$ and some $B \supset A$, where $A, B$ are both $(\mathcal{M}, 1)$-neat, and use the $\left(<\aleph_{0},<\aleph_{0},<\kappa^{+}\right)$-dap on $K$ to find the amalgam of $\left(A, B, \mathcal{A}_{\alpha-1}\right)$; let $\mathcal{A}_{\alpha}$ be that amalgam, and write $\mathcal{A}_{\alpha}=\operatorname{amalgam}\left(A, B, \mathcal{A}_{\alpha-1}\right)$; and

(5) for all $\alpha<\kappa^{+}$and for all $A \subset \mathcal{A}_{\alpha}$ and $B \supset A$, where $A, B$ are both $(\mathcal{M}, 1)$-neat, there exist $\kappa^{+}$-many $\beta>\alpha$ such that $\mathcal{A}_{\beta}=\operatorname{amalgam}(A, B$, $\left.\mathcal{A}_{\beta-1}\right)$.

This decomposition is possible since for all $\alpha$ there are $\left(<\kappa^{+}\right)$-many $(\mathcal{M}, 1)$-neat $A \subset \mathcal{A}_{\alpha}$ and $\kappa$-many (nonisomorphic) $(\mathcal{M}, 1)$-neat $B \supset A$, and we mentioned already that for every pair $(A, B)$ there are $\kappa^{+}$-many disjoint copies of $B$ in $\mathcal{A}$.

Using this decomposition we can make $\mathcal{A}^{2}$ into an $\left(\mathcal{M}, \kappa^{+}\right)$-full structure, that is, assign to every $x \in \mathcal{A} \backslash\left(\mathcal{M} \cup \kappa^{+}\right)$some unique $k \in \kappa^{+}$such that $P(k, x)$. The property that needs work is property (2) of Definition 3.6. Proceed by induction on $\alpha$, and assume that for all $x \in A_{\alpha}$ there exists $k \in \kappa^{+}$such that $P(k, x)$. We need to extend $P$ on $\mathcal{A}_{\alpha+1}$ too. By definition $\mathcal{A}_{\alpha+1}=\operatorname{amalgam}\left(A, B, \mathcal{A}_{\alpha}\right)$, for some $(\mathcal{M}, 1)$-neat $A \subset \mathcal{A}_{\alpha}$ and $B \supset A$. In particular, there exists some embedding $i: B \rightarrow \mathcal{A}_{\alpha+1}$ such that $\left.i\right|_{A}=i d$ and $i[B] \cap \mathcal{A}_{\alpha}=A$. By the inductive hypothesis and since $A \subset \mathcal{A}_{\alpha}, A$ is also an $\left(\mathcal{M}, \kappa^{+}\right)$-neat structure, and there are $\kappa^{+}$-many different ways to make $B$ into an $\left(\mathcal{M}, \kappa^{+}\right)$-neat structure. Fix such a way; that is, fix a partition of $B$ by elements in $\kappa^{+}$, and let for all $i(b) \in i[B] \backslash A$ and all $k \in \kappa^{+}$,

$$
P(k, i(b)) \quad \text { if and only if } \quad P(k, b) .
$$

Since by (5) of the definition of the $\mathcal{A}_{\alpha}$ 's, the same $A, B$ appear $\kappa^{+}$-many times, we can ensure that every possible partitioning of $B$ (there are $\kappa^{+}$-many of them) is used 
at least once. Finally, for all elements (if any) $x \in \mathcal{A}_{\alpha+1} \backslash\left(\mathcal{A}_{\alpha} \cup i[B]\right)$ assign $P(k, x)$ for some arbitrary $k \in \kappa^{+}$. It is not hard to see that the definition was cooked in such a way that $\mathcal{A}$ satisfies property (2) of Definition 3.6. and the result follows.

Some observations:

(a) Part (a) of Theorem 3.18 is the converse of part (b) when $\lambda=\kappa^{+}$. In particular, there exists an $\left(\mathcal{M}, \kappa^{+}\right)$-full structure of size $\kappa^{+}$if and only if there exists an $(\mathcal{M}, 1)$-full structure of size $\kappa^{+}$such that the collection $K$ of all $(\mathcal{M}, 1)$-happy substructures satisfies the $\left(<\boldsymbol{\aleph}_{0},<\boldsymbol{\aleph}_{0},<\kappa^{+}\right)$-dap.

(b) The theorem holds true if we replace $(\mathcal{M}, 1)$-full by $(\mathcal{M}, \mu)$-full, where $\mu \leq \lambda$, in part (a). Similarly, we can replace $(\mathcal{M}, 1)$-full and $(\mathcal{M}, 1)$-happy by $(\mathcal{M}, \mu)$-full and $(\mathcal{M}, \mu)$-happy, respectively, in part (b), where $\mu<\kappa^{+}$.

(c) In part (b) we exploited the full strength of the $\left(<\boldsymbol{\aleph}_{0},<\boldsymbol{\aleph}_{0},<\kappa^{+}\right)$-dap. It is open whether the converse of part (a) holds, that is, whether we can use the $\left(<\aleph_{0},<\aleph_{0},<\lambda\right)$-dap to prove the existence of an $(\mathcal{M}, \lambda)$-full structure of size $\kappa^{+}$, where $\lambda<\kappa^{+}$.

(d) Property (2) in the definition of an $(\mathcal{M}, \mathcal{N})$-full structure (see Definition 3.6) is equivalent to the $\left(<\boldsymbol{\aleph}_{0},<\boldsymbol{\aleph}_{0},<\boldsymbol{\aleph}_{0}\right)$-dap for the class of all $(\mathcal{M}, \mathcal{N})$-happy structures.

Theorem 3.19 If $\kappa \in \mathcal{C} \mathscr{H}_{\omega_{1}, \omega}$, then at least one of the following is the case (see Definitions 1.3 and 1.4):

1. $\kappa^{+} \in \mathscr{H}^{\mathcal{C}} \mathscr{H}_{\omega_{1}, \omega}$, or

2. $\left(\kappa^{+}, \kappa\right) \in \mathscr{H}^{\mathcal{C}} \mathscr{H}_{\omega_{1}, \omega}$, or

3. $\kappa \in \mathscr{H} \mathcal{H} \mathscr{\omega}_{1}, \omega$.

Proof Let $\varphi$ be a complete sentence that witnesses $\kappa \in \mathcal{C} \mathscr{H}_{\omega_{1}, \omega}$. In particular, if $\mathcal{M}$ is any model of $\varphi,|\mathcal{M}| \leq \kappa$ and by Corollary 3.12 there are no $(\mathcal{M}, \mathcal{N})$-full structures of size at least $\kappa^{++}$. On the other hand for any $\mathcal{M} \models \varphi$ such that $|\mathcal{M}|=\kappa$ and any $\mathcal{N}$ of size $\kappa$, there exists an $(\mathcal{M}, \mathcal{N})$-full structure of size $\kappa$ by Lemma 3.8.

Let $M_{0}$ be the (unique) countable model of $\varphi$, let $\mathcal{N}_{0}$ be a model of size $\boldsymbol{\aleph}_{0}$ such that $\mathscr{L}\left(\mathcal{N}_{0}\right)=\emptyset$, and let $\mathcal{A}$ be a $\left(\mathcal{M}_{0}, \mathcal{N}_{0}\right)$-full structure of size $\boldsymbol{\aleph}_{0}$. By Lemma 3.10, $\mathcal{A}$ is unique (up to isomorphism), and let $\psi$ be its Scott sentence. From Lemma 3.13, $\mathcal{N}_{0}$ is completely homogeneous for $\mathcal{A}$. By Observation 3.7(d), any model of $\psi$ is ( $\mathcal{M}, \lambda)$-full for some $\mathcal{M}$ model of $\varphi$ and some $\aleph_{0} \leq \lambda \leq \kappa^{+}$. The proof splits into three cases.

Case I. There is no $(\mathcal{M}, \mathcal{N})$-full structure of size $\kappa^{+}$, where $\mathcal{M}$ is a model of $\varphi$.

We observed above that the existence of $(\mathcal{M}, \mathcal{N})$-full structures of size $\kappa$ follows by Lemma 3.8. Therefore $\psi$ witnesses that $\kappa \in \mathscr{H}_{\mathcal{C}} \mathscr{H}_{\omega_{1}, \omega}$.

Case II. There exists an $\left(\mathcal{M}, \kappa^{+}\right)$-full structure of size $\kappa^{+}$.

In this case $\psi$ witnesses that $\kappa^{+} \in \mathscr{H}^{\mathcal{C}} \mathscr{H}_{\omega_{1}, \omega}$.

Case III. There exists an $(\mathcal{M}, \lambda)$-full structure of size $\kappa^{+}$with $\lambda \leq \kappa$ but no $\left(\mathcal{M}, \kappa^{+}\right)$-full structure of size $\kappa^{+}$.

In this case $\psi$ witnesses that $\left(\kappa^{+}, \kappa\right) \in \mathscr{H}^{\mathcal{C}} \mathscr{H}_{\omega_{1}, \omega}$ since the homogeneous part of the model that corresponds to $\mathcal{N}_{0}$ cannot have size $\kappa^{+}$, while by Lemma 3.8, it can have size equal to $\kappa$. 
Notes (a) Although Cases I, II, and III in the proof of Theorem 3.19 are exclusive to one another, (1), (2), and (3) in the statement of Theorem 3.19 need not be exclusive to one another. (b) It seems to the author that for a particular $\kappa$ it is independent of ZFC whether Case I or Case II or Case III holds, but we do not have a proof for that.

Lemma 3.20 Let $\kappa$ be an infinite cardinal, and let $\mathcal{M}$ be a model of size $\kappa$. If $\kappa^{\omega}=\kappa$, then there is no $(\mathcal{M}, \mathcal{N})$-full structure of size $\kappa^{+} .^{3}$

Proof Assume that there is an $\mathcal{A}$ that is $(\mathcal{M}, \mathcal{N})$-full and has size $\kappa^{+}$. Then for every $a \in \mathcal{A} \backslash(\mathcal{M} \cup \mathcal{N})$, let $f_{a}$ be the function given by

$$
f_{a}(b)=m \quad \text { if and only if } \quad S(m, a, b) .
$$

This defines a family of functions $\mathcal{F}=\left\{f_{a} \mid a \in \mathcal{A} \backslash(\mathcal{M} \cup \mathcal{N})\right\}$ that has size $\kappa^{+}$. Now, let $\mathcal{A}_{0} \subset \mathcal{A} \backslash \mathcal{M}$ be a subset of cardinality $\omega$, and consider the restrictions $\left.f_{a}\right|_{\mathcal{A}_{0}}$. By assumption there are $\left(\kappa^{\omega}=\kappa\right)$-many possible distinct such functions. Since $|\mathcal{F}|=\kappa^{+}$, there exists a subset $X \subset \mathcal{F}$ of size $\kappa^{+}$such that for all $f_{a}, f_{b} \in X, f_{a}\left|\mathcal{A}_{0}=f_{b}\right| \mathcal{A}_{0}$. On the other hand, for any $a, b \in \mathcal{A} \backslash(\mathcal{M} \cup \mathcal{N})$, by $\left(* 2^{\mathcal{M}, \mathcal{N}}\right)$ there exist only finitely many $b_{0}, b_{1}, \ldots, b_{k-1}$ such that for any $m \in \mathcal{M}, S\left(m, a, b_{i}\right) \Leftrightarrow S\left(m, b, b_{i}\right)$. In particular, $f_{a}(x)=f_{b}(x)$ if and only if $x \in\left\{b_{0}, b_{1}, \ldots, b_{k-1}\right\}$, which contradicts the conclusion that any two $f_{a} \neq f_{b} \in X$ agree on $\mathcal{A}_{0}$, a set of size $\omega$.

Theorem 3.21 If $\kappa \in \mathcal{C} \mathscr{H}_{\omega_{1}, \omega}$ and $\kappa^{\omega}=\kappa$, then $\kappa \in \mathscr{H}^{\mathcal{C}} \mathscr{H}_{\omega_{1}, \omega}$.

Proof The theorem is proved by the previous lemma and Case I of (the proof of) Theorem 3.19.

It is consistent that all limit cardinals of uncountable cofinality satisfy $\kappa^{\omega}=\kappa$ (under GCH for instance). In this case, by Theorem 3.21, if $\kappa \in \mathcal{C}_{\omega_{\omega_{1}, \omega}}$ and cf $(\kappa)>\omega$, then $\kappa \in \mathscr{H}_{\mathcal{C}} \mathscr{H}_{\omega_{1}, \omega}$. Hence, it is consistent that infinite cardinal $\kappa$ is in $\mathcal{C} \mathscr{H}_{\omega_{1}, \omega} \backslash \mathscr{H} \mathcal{H} \mathscr{H}_{\omega_{1}, \omega}$ only if $\kappa$ has cofinality $\omega$.

Conjecture 1 For an infinite cardinal $\kappa, \kappa \in \mathcal{C} \mathscr{H}_{\omega_{1}, \omega} \backslash \mathscr{H}^{\mathcal{C}} \mathscr{H}_{\omega_{1}, \omega}$ if and only if $c f(\kappa)=\omega$.

\section{Powers}

Here we investigate powers of the form $\lambda^{\kappa}$, where $\lambda, \kappa$ are characterizable. The main theorem is Theorem 4.6: If $\lambda$ is in $\mathcal{C} \mathscr{H}_{\omega_{1}, \omega}$, then $\lambda^{\omega}$ is in $\mathscr{H} \mathcal{H} \mathscr{H}_{\omega_{1}, \omega}$. The idea behind the construction is similar to Malitz's proof that $2^{\omega} \in \mathscr{H}^{\mathcal{C}} \mathscr{H}_{\omega_{1}, \omega}$ in [6].

Theorem 4.1 (Baumgartner [1]) $\quad$ If $\kappa \in \mathscr{H}^{\mathcal{C}} \mathscr{H}_{\omega_{1}, \omega}$, then $2^{\kappa} \in \mathscr{H}^{\mathcal{C}} \mathscr{H}_{\omega_{1}, \omega}$.

In particular, if $\lambda \leq \kappa$ and $\kappa \in \mathscr{H}^{\mathcal{C}} \mathscr{H}_{\omega_{1}, \omega}$, then $\lambda^{\kappa} \in \mathscr{H}^{\mathcal{C}} \mathscr{H}_{\omega_{1}, \omega}$.

We can also adapt the proof of Theorem 4.1 to prove the following.

Theorem 4.2 If $\kappa, \lambda$ are infinite cardinals such that $\lambda \leq \kappa \leq 2^{\lambda}$ and $(\kappa, \lambda) \in$ $\mathscr{H}^{\mathcal{C}} \mathscr{H}_{\omega_{1}, \omega}$, then $2^{\lambda} \in \mathscr{H}^{\mathcal{C}} \mathscr{H}_{\omega_{1}, \omega}$.

An easy induction gives the following.

Lemma 4.3 For every $\alpha<\omega_{1}$, there is a sentence $\sigma_{\alpha}$ in a language that contains the binary symbol $<$ such that

$$
M \models \sigma_{\alpha} \quad \text { if and only if } \quad\left(M ;<^{M}\right) \cong(\alpha ; \epsilon) .
$$

The first goal is to prove the following theorem. 
Theorem 4.4 If $\lambda \in \mathscr{H}^{\mathcal{C}} \mathscr{H}_{\omega_{1}, \omega}$, then $\lambda^{\omega} \in \mathcal{C} \mathscr{H}_{\omega_{1}, \omega}$.

Proof First note that $\lambda$ is homogeneously characterizable, while $\lambda^{\omega}$ is just characterizable. Since $\lambda \in \mathscr{H}^{\mathcal{C}} \mathscr{H}_{\omega_{1}, \omega}$, there exists a complete sentence $\varphi_{\lambda}$ in a language that contains a predicate symbol $P_{\lambda}$ such that $\left(\varphi_{\lambda}, P_{\lambda}\right)$ homogeneously characterize $\lambda$ (see Definition 1.3). That is,

- $\varphi_{\lambda}$ does not have models of power greater than $\lambda$;

- if $\mathcal{M}$ is the (unique) countable model of $\varphi_{\lambda}$, then $P_{\lambda}$ is infinite and completely homogeneous for $\mathcal{M}$; and

- there is a model $\mathcal{A}$ of $\varphi_{\lambda}$ such that $P_{\lambda}^{\mathcal{A}}$ has cardinality $\lambda$.

Now let $\mathscr{L}$ be the signature that contains all symbols in $\varphi_{\lambda}$ as well as new symbols $K(\cdot), F(\cdot), V(\cdot, \cdot), R(\cdot, \cdot), E(\cdot, \cdot, \cdot)$, and $<$. The idea is to build a rooted tree of height $\omega$ where at every level we allow at most $\lambda$-splitting. $V(\cdot, \cdot)$ captures the set of vertices, with $V(n, \cdot)$ being the set of vertices of height $n<\omega$. For vertices $x, y, R(x, y)$ holds if and only if $x$ and $y$ are adjacent vertices, with $y$ being a descendant of $x$ (i.e., there is some $n$ such that $V(n, x), V(n+1, y)$, and $x, y$ are connected). $F(\cdot)$ will capture the set of maximal branches through the tree. If $F(f)$, we will think of $f$ as a function with domain $\omega$ and $f(n)$ will be in $V(n, \cdot) . E(f, n, y)$ indicates that $f(n)=y$, and we will just write $f(n)=y$ for short.

The difficulty is to express $\lambda$-splitting, and this is where we use the full power of the fact that $\lambda$ is in $\mathscr{H} \mathcal{C} \mathscr{H}_{\omega_{1}, \omega}$. To every vertex $y$, we assign a structure $M_{y}=M(y, \cdot)$, and we stipulate that $M_{y}$ together with the set of all the descendants of $y$ satisfy $\varphi_{\lambda}$. Thus, they must have size at most $\lambda$. It takes some argument to prove that this yields a complete sentence (see Claim 3).

Consider the conjunction of the following sentences.

(1) $(K ;<) \cong(\omega ; \in)$. This we can say by using Lemma 4.3.

So, we will freely write $0,1, \ldots, n, n+1, \ldots$ for the elements of $K$.

(2) Let $V=\bigcup_{n \in K} V(n, \cdot)$. Then $K \cup F \cup V \cup_{y \in V} M(y, \cdot)$ partition the whole space. All of them are infinite, except $V(0, \cdot)$.

(3) (a) $V(n, y)$ implies that $n \in K$. Write $y \in V(n)$ for $V(n, y)$.

(b) For $n \neq m \in K, V(n) \cap V(m)=\emptyset$.

(c) $V(0)=\{a\}$, where $a$ can be anything. This will be the root of the tree.

(4) (a) If $F(f)$, we will write $f \in F$.

(b) If $E(f, n, y)$, then $f \in F, n \in K$ and $y \in V(n)$. We will write $f(n)=y$ instead of $E(f, n, y)$.

(c) We have $\forall n \in K \forall f \in F \exists$ ! $y \in V(n), f(n)=y$. That is, every $f \in F$ is a function with domain $\omega$ and such that $f(n) \in V(n)$, for every $n$.

(d) We have $\forall f \neq g \in F \exists n \in K, f(n) \neq g(n)$.

(5) (a) We have $\forall y, z(R(y, z) \Rightarrow \exists ! n \in K(y \in V(n) \wedge z \in V(n+1)))$.

(b) For all $y$, the set $\{z \mid R(y, z)\}$ is infinite.

(c) We have $\forall n \in K \forall z \in V(n+1) \exists ! y R(y, z)$. By (a), this $y$ must be in $V(n)$.

(d) We have $\forall y_{1} \neq y_{2} \forall z\left(R\left(y_{1}, z\right) \Rightarrow \neg R\left(y_{2}, z\right)\right)$, that is, $R\left(y_{1}, \cdot\right)$ and $R\left(y_{2}, \cdot\right)$ are disjoint.

(e) We have $\forall n \in K \forall f \in F(R(f(n), f(n+1)))$.

(f) We have $\forall n \in K \forall y_{0}, y_{1}, \ldots, y_{n},\left(y_{0}=a \bigwedge_{i \leq n} R\left(y_{i}, y_{i+1}\right)\right) \Rightarrow$ $\left.\exists^{\infty} f \in F\left(\bigwedge_{i \leq n} f(i)=y_{i}\right)\right)$, that is, every finite "branch" can be extended to a maximal branch in infinitely many ways. 
(6) (a) We have $\forall y \in V M(y, \cdot) \cup R(y, \cdot) \models \varphi_{\lambda}$.

(b) We have $\forall y \in V \forall x \in M(y, \cdot) \cup R(y, \cdot),\left(P_{\lambda}(x) \Leftrightarrow R(y, x)\right)$.

The above two sentences express the fact that $M(y, \cdot)$ together with $R(y, \cdot)$ satisfy $\varphi_{\lambda}$ and $R(y, \cdot)$ is the part of the model given by the homogeneous predicate $P_{\lambda}$. This restricts the size of $R(y, \cdot)$ to at most $\lambda$. Since $R(y, \cdot)$ is the homogeneous part of the model, in the countable case, every permutation of it can be extended to an automorphism of the whole model. We will use this in what follows.

The goal now is to show that a structure that satisfies (1)-(6) characterizes $\lambda^{\omega}$.

Claim $1 \quad$ If $\mathcal{M} \models(1)-(6)$, then $|\mathcal{M}| \leq \lambda^{\omega}$.

Proof First we prove by induction on $n \in K$ that $|V(n)| \leq \lambda^{n}$.

For $n=0,|V(0)|=|\{a\}|=1=\lambda^{0}$. Assume that $|V(n)| \leq \lambda^{n}$, and let $z \in V(n+1)$. By $5(\mathrm{a})$, there is a unique $y \in V(n)$ such that $R(y, z)$. So,

$$
V(n+1)=\bigcup_{y \in V(n)} R(y, \cdot)
$$

By $5(\mathrm{~d})$, all these $R(y, \cdot)$ are disjoint, and by (6) all of them have size at most $\lambda$. Thus,

$$
|V(n+1)|=\sum_{y \in V(n)}|R(y, \cdot)| \leq \sum_{y \in V(n)} \lambda=|V(n)| \cdot \lambda \leq \lambda^{n+1},
$$

as we want. Therefore $\left|\bigcup_{n \in K} V(n)\right| \leq \lambda^{<\omega}=\lambda$.

Since, for every $f \in F, f$ is a function from $K$ to $\bigcup_{n \in K} V(n),|F| \leq \lambda^{\omega}$. Also, for every $y, M(y, \cdot)$ has size at most $\lambda$. So,

$$
|\mathcal{M}| \leq|K|+|F|+\left|\bigcup_{n \in K} V(n)\right|+\left|\bigcup_{y} M(y, \cdot)\right| \leq \omega+\lambda^{\omega}+\lambda+\lambda \cdot \lambda=\lambda^{\omega} .
$$

Claim 2 There is $\mathcal{M} \models(1)-(6)$ and $|\mathcal{M}|=\lambda^{\omega}$.

Proof Take the full $\lambda$-tree $(T, R)$ of height $\omega$, with $f \in F$ being its maximal branches. The rest follows.

Claim 3 If $\mathcal{M}_{1}, \mathcal{M}_{2}$ are both countable models of (1)-(6), then there is an isomorphism $i: \mathcal{M}_{1} \cong \mathcal{M}_{2}$.

Proof First of all we observe that $\left(K\left(\mathcal{M}_{1}\right) ;<\right) \cong(\omega ; \epsilon) \cong\left(K\left(\mathcal{M}_{2}\right) ;<\right)$, so that we do not have to worry about $K$.

Subclaim 1 For all $n \in K$ and for all $y \in V(n)$, there is $f \in F$ with $f(n)=y$.

Proof By 5(c) and by induction on $n$, if $y \in V(n)$, there is a (unique) sequence $y_{0}=a, y_{1}, \ldots, y_{n-1}, y_{n}=y$ such that $\bigwedge_{i<n} R\left(y_{i}, y_{i+1}\right)$. By 5 (f) there is some $f$ that "extends" this sequence; that is, for all $i \leq n, f(i)=y_{i}$. In particular, $f(n)=y$.

Subclaim 2 For all $f_{1} \neq f_{2} \in F$, there exists $n>0$ such that $\forall m<n\left(f_{1}(m)=\right.$ $\left.f_{2}(m)\right)$ and $\forall m^{\prime} \geq n\left(f_{1}\left(m^{\prime}\right) \neq f_{2}\left(m^{\prime}\right)\right)$. We call this $n$ the splitting point of $f_{1}, f_{2}$, $n=s \cdot p\left(f_{1}, f_{2}\right)$. 
Proof Since $f_{1} \neq f_{2}$, there exists $n^{\prime}$ so that $f_{1}\left(n^{\prime}\right) \neq f_{2}\left(n^{\prime}\right)$. Let $n$ be the least such. By 3(c), $n>0$. Assume also that there exists $m^{\prime}>n$ such that $f_{1}\left(m^{\prime}\right)=f_{2}\left(m^{\prime}\right)$. Again, let $m$ be the least such. Then, $y_{1}=f_{1}(m-1) \neq y_{2}=$ $f_{2}(m-1)$. Therefore, by $5(\mathrm{~d}), R\left(y_{1}, \cdot\right) \cap R\left(y_{2}, \cdot\right)=\emptyset$, and by $5(\mathrm{e}), f_{1}(m) \in R\left(y_{1}, \cdot\right)$ and $f_{2}(m) \in R\left(y_{2}, \cdot\right)$ with $f_{1}(m)=f_{2}(m)$, a contradiction.

We will, now, define $i: \mathcal{M}_{1} \cong \mathcal{M}_{2}$ by induction, so that, if $F\left(\mathcal{M}_{1}\right)=\left\{f_{1}, \ldots\right.$, $\left.f_{n}, \ldots\right\}$ and $F\left(\mathcal{M}_{2}\right)=\left\{g_{1}, \ldots, g_{n}, \ldots\right\}$, then $f_{n}$ (together with all the values $\left.y=f_{n}(m)\right)$ is included in the domain of $i$ at step $2 n$, while at step $2 n+1$ we make sure to include $g_{n}$ (and all the corresponding values). We do this in a way that the relations $R, V$ and the structures $M(y, \cdot)$ are preserved.

Step 2n: Say that $i$ has been defined on

$$
X=\left\{f_{1}, \ldots, f_{n-1}, i^{-1}\left(g_{1}\right), \ldots, i^{-1}\left(g_{n-1}\right)\right\}
$$

so far and that $f_{n}$ is not in $X$. Let $f \in X$ such that

$$
m=s \cdot p\left(f, f_{n}\right) \geq \operatorname{s.p}\left(f^{\prime}, f_{n}\right), \quad \text { for all } f^{\prime} \in X .
$$

By inductive assumption, all the images (under $i$ ) of the values $y_{0}=f(0)=f_{n}(0)$, $\ldots, y_{m-1}=f(m-1)=f_{n}(m-1)$ have already been defined. Let $z_{j}=i\left(y_{j}\right)$, for $j<m$. In $\mathcal{M}_{2}$ it holds by 5(e) that $\bigwedge_{j<m-1} R\left(z_{j}, z_{j+1}\right)$. By 5(b), there are infinitely many values in $R\left(z_{m-1}, \cdot\right)$. Choose one of them, $z_{m}$, that is different than all of $i\left(f_{1}\right)(m), \ldots, i\left(f_{n-1}\right)(m)$ and $g_{1}(m), \ldots, g_{n-1}(m)$, and set $i\left(f_{n}(m)\right)=z_{m}$. By 5(f), there is a function $g \in F\left(\mathcal{M}_{2}\right)$ that "extends" $\left(z_{0}, \ldots, z_{m}\right)$. Let $i\left(f_{n}\right)=g$, and for $m^{\prime}>m$, let $i\left(f_{n}\left(m^{\prime}\right)\right)=g\left(m^{\prime}\right)$. Obviously $g$ is different than all of $i\left(f_{1}\right), \ldots, i\left(f_{n-1}\right)$ and $g_{1}, \ldots, g_{n-1}$, and by $4(\mathrm{c})$ and $5, R, V$ are preserved.

Step $2 n+1$ : Similarly.

Eventually, we will have included in the domain of $i$ the whole $F\left(\mathcal{M}_{1}\right)$ and in the range of $i$ all of $F\left(\mathcal{M}_{2}\right)$. By Subclaim 1, this also means that $V\left(n, \mathcal{M}_{1}\right), V\left(n, \mathcal{M}_{2}\right)$ are included in the domain and the range of $i$, respectively. As we mentioned, $R$ and $V$ are preserved and $i$ becomes an isomorphism given that we can extend $i$ on each of the structures $M(y, \cdot)$.

To this end, let $y \in \mathcal{M}_{1}$ and $z=i(y)$. By $6(\mathrm{a}), M\left(y, \mathcal{M}_{1}\right) \cup R\left(y, \mathcal{M}_{1}\right) \models \varphi_{\lambda}$ and $M\left(z, \mathcal{M}_{2}\right) \cup R\left(z, \mathcal{M}_{2}\right) \models \varphi_{\lambda}$. By the completeness assumption on $\varphi_{\lambda}$ and since both $\mathcal{M}_{1}, \mathcal{M}_{2}$ are countable, there is $j: M\left(y, \mathcal{M}_{1}\right) \cup R\left(y, \mathcal{M}_{1}\right) \cong M\left(z, \mathcal{M}_{2}\right) \cup R\left(z, \mathcal{M}_{2}\right)$. The problem is that $i, j$ may not agree on $R\left(y, \mathcal{M}_{1}\right)$. In either case, there exists a permutation $\pi$ of $R\left(y, \mathcal{M}_{1}\right)$ such that for all $y^{\prime} \in R\left(y, \mathcal{M}_{1}\right)$,

$$
j\left(\pi\left(y^{\prime}\right)\right)=i\left(y^{\prime}\right) .
$$

By $P_{\lambda}$ being a homogeneous predicate, every such $\pi$ will induce an automorphism of $M\left(y, \mathcal{M}_{1}\right) \cup R\left(y, \mathcal{M}_{1}\right)$, call it $j_{\pi}$. Then for every $y^{\prime} \in R\left(y, \mathcal{M}_{1}\right)$,

$$
j\left(j_{\pi}\left(y^{\prime}\right)\right)=j\left(\pi\left(y^{\prime}\right)\right)=i\left(y^{\prime}\right) .
$$

So, we can extend $i$ on the whole of $M\left(y, \mathcal{M}_{1}\right) \cup R\left(y, \mathcal{M}_{1}\right)$ by

$$
i\left(y^{\prime}\right)=j\left(j_{\pi}\left(y^{\prime}\right) .\right.
$$

Since $j$ is onto $M\left(z, \mathcal{M}_{2}\right) \cup R\left(z, \mathcal{M}_{2}\right)$, we conclude that $i: \mathcal{M}_{1} \cong \mathcal{M}_{2}$.

The three previous claims complete the proof. 
Note Obviously, if we could characterize $\omega_{1}$ by an $\mathscr{L}_{\omega_{1}, \omega}$-sentence, then we would also get characterizability of $\lambda^{\omega_{1}}$, etc. But this is not possible. The inability to characterize well-founded linear orderings also makes the obvious attempt to characterize $\lambda^{\kappa}$ fail.

Theorem 4.5 If $\lambda^{\omega}>\lambda$ and $\left(\lambda^{+}, \lambda\right) \in \mathscr{H}^{\mathcal{C}} \mathscr{H}_{\omega_{1}, \omega}$ (see Definition 1.4), then $\lambda^{\omega} \in \mathcal{C} \mathscr{H}_{\omega_{1}, \omega}$.

Proof Work as in the proof of Theorem 4.4; but Claim 1 is now slightly different. Under the new assumption, for every vertex $y, M_{y}=M(y, \cdot)$ may have size up to $\lambda^{+}$. On the other hand, by the definition of $\left(\lambda^{+}, \lambda\right) \in \mathscr{H}^{\mathcal{C}} \mathscr{H}_{\omega_{1}, \omega}$, the homogeneous part has size at most $\lambda$. In particular, for every $y, R(y, \cdot)$ has size at most $\lambda$ and the $\lambda$-splitting is not affected. Since $\lambda^{\omega} \geq \lambda^{+}$, Claim 1 holds true.

We can actually do a bit better.

Theorem 4.6 If $\lambda \in \mathcal{C} \mathscr{H}_{\omega_{1}, \omega}$, then $\lambda^{\omega} \in \mathscr{H}^{\mathcal{C}} \mathscr{H}_{\omega_{1}, \omega}$.

Proof First observe that $\lambda$ is now characterizable and that $\lambda^{\omega}$ is homogeneous characterizable. So, the assumption of the theorem is slightly weaker than Theorem 4.4, and the conclusion is slightly stronger.

Applying Theorem 3.21 to $\lambda^{\omega}$, we conclude that $\lambda^{\omega} \in \mathscr{H}^{\mathcal{C}} \mathscr{H}_{\omega_{1}, \omega}$ if and only if $\lambda^{\omega} \in \mathcal{C} \mathscr{H}_{\omega_{1}, \omega}$. So, it suffices to prove that $\lambda^{\omega} \in \mathcal{C} \mathscr{H}_{\omega_{1}, \omega}$. If $\lambda$ is in $\mathscr{H} \mathcal{H} \mathscr{H}_{\omega_{1}, \omega}$, then we can use Theorem 4.4. So, assume that $\lambda$ is not in $\mathscr{H}^{\mathcal{C}} \mathscr{H}_{\omega_{1}, \omega}$ for the rest of the proof. In particular, by Theorem $3.21, \lambda^{\omega}>\lambda$. We split the proof into two cases given by cases (1) and (2) of Theorem 3.19:

(1) $\lambda^{+} \in \mathscr{H}^{\mathcal{C}} \mathscr{H}_{\omega_{1}, \omega}$ : by the Hausdorff formula,

$$
\left(\lambda^{+}\right)^{\omega}=\lambda^{+} \cdot \lambda^{\omega}=\lambda^{\omega}
$$

and use Theorem 4.4.

(2) $\left(\lambda^{+}, \lambda\right) \in \mathscr{H}^{C} \mathscr{H}_{\omega_{1}, \omega}$ : then use Theorem 4.5.

Corollary 4.7 If $\kappa$ is an infinite cardinal and $\lambda^{\kappa} \in \mathcal{C} \mathscr{H}_{\omega_{1}, \omega}$, then $\lambda^{\kappa} \in$ $\mathscr{H} \mathcal{H} \mathscr{H}_{1}, \omega$.

Corollary 4.8 $\quad \mathcal{H} \mathscr{H}_{\omega_{1}, \omega}$ and $\mathscr{H}^{\mathcal{C}} \mathscr{H}_{\omega_{1}, \omega}$ are both closed under countable products.

Proof We have

$$
\prod_{n} \aleph_{\alpha_{n}}=\left(\sup _{n} \aleph_{\alpha_{n}}\right)^{\aleph_{0}}
$$

Theorem 4.9 If $\boldsymbol{\aleph}_{\alpha}^{\boldsymbol{\aleph}_{\beta}} \in \mathcal{C} \mathscr{H}_{\omega_{1}, \omega}$, then for all $\gamma<\omega_{1}$,

$$
\boldsymbol{\aleph}_{\alpha+\gamma}^{\boldsymbol{\aleph}_{\beta}} \in \mathscr{H} \mathcal{H} \mathscr{H}_{\omega_{1}, \omega},
$$

that is, if one power of $\aleph_{\alpha}$ is in $\mathcal{C} \mathscr{H}_{\omega_{1}, \omega}$, the same is true for the powers of a whole cluster of cardinals.

Proof First observe that by Corollary 4.7 , if $\boldsymbol{\aleph}_{\alpha+\gamma}^{\boldsymbol{\aleph}_{\beta}}$ is in $\mathcal{C} \mathscr{H}_{\omega_{1}, \omega}$, then it is also in $\mathscr{H}^{\mathcal{C}} \mathscr{H}_{\omega_{1}, \omega}$. So, we do not worry about homogeneity. We proceed by induction on $\gamma$.

If $\gamma=\gamma_{1}+1$, a successor ordinal, then by the Hausdorff formula again

$$
\boldsymbol{\aleph}_{\alpha+\gamma_{1}+1}^{\boldsymbol{\aleph}_{\beta}}=\boldsymbol{\aleph}_{\alpha+\gamma_{1}+1} \cdot \boldsymbol{\aleph}_{\alpha+\gamma_{1}}^{\boldsymbol{\aleph}_{\beta}} .
$$


The second factor is in $\mathscr{H}^{\mathcal{C}} \mathscr{H}_{\omega_{1}, \omega}$ by inductive hypothesis and if $\boldsymbol{\aleph}_{\alpha+\gamma_{1}+1}^{\boldsymbol{\aleph}_{\beta}}=\boldsymbol{\aleph}_{\alpha+\gamma_{1}}^{\boldsymbol{\aleph}_{\beta}}$, we can conclude that $\boldsymbol{\aleph}_{\alpha+\gamma_{1}+1}^{\aleph_{\beta}}$ is also in $\mathscr{H}^{\mathcal{C}} \mathscr{H}_{\omega_{1}, \omega}$. If on the other hand $\boldsymbol{\aleph}_{\alpha+\gamma_{1}+1}^{\aleph_{\beta}}>\boldsymbol{\aleph}_{\alpha+\gamma_{1}}$, then it must be the case that $\boldsymbol{\aleph}_{\alpha+\gamma_{1}+1}=\boldsymbol{\aleph}_{\alpha+\gamma_{1}+1}$ and $\boldsymbol{\aleph}_{\alpha+\gamma_{1}}^{\boldsymbol{\aleph}_{\beta}}=\boldsymbol{\aleph}_{\alpha+\gamma_{1}}$. By the inductive hypothesis, $\boldsymbol{\aleph}_{\alpha+\gamma_{1}} \boldsymbol{\aleph}_{\beta}$ is in $\mathcal{C} \mathscr{H}_{\omega_{1}, \omega}$, and the same is true for $\boldsymbol{\aleph}_{\alpha+\gamma_{1}}$. We conclude that $\boldsymbol{\aleph}_{\alpha+\gamma_{1}+1} \in \mathcal{C} \mathscr{H}_{\omega_{1}, \omega}$ by Theorem 3.1.

If $\gamma=\lambda, \gamma \neq 0$, a countable limit ordinal, then $c f(\alpha+\gamma)=c f(\gamma)=\omega$ and $\aleph_{\alpha+\gamma}=\sup _{n} \aleph_{\alpha+\gamma_{n}}$, for an increasing sequence of $\gamma_{n}$ 's. Then,

$$
\boldsymbol{\aleph}_{\alpha+\gamma}^{\aleph_{\beta}}=\left(\lim _{n} \boldsymbol{\aleph}_{\alpha+\gamma_{n}}^{\boldsymbol{\aleph}_{\beta}}\right)^{c f(\alpha+\gamma)}=\left(\lim _{n} \boldsymbol{\aleph}_{\alpha+\gamma_{n}}^{\boldsymbol{\aleph}_{\beta}}\right)^{\boldsymbol{\aleph}_{0}}=\prod_{n}\left(\boldsymbol{\aleph}_{\alpha+\gamma_{n}}^{\boldsymbol{\aleph}_{\beta}}\right) \in \mathcal{H} \mathcal{C} \mathscr{H}_{\omega_{1}, \omega},
$$

by the inductive assumption and Corollary 4.8 .

In particular, for $\alpha=0$ we conclude that

$$
\boldsymbol{\aleph}_{\gamma}^{\boldsymbol{\aleph}_{\beta}} \in \mathscr{H}^{\mathcal{C}} \mathscr{H}_{\omega_{1}, \omega} \quad \text { for all } \gamma<\omega_{1} \quad \text { if and only if } \quad 2^{\boldsymbol{\aleph}_{\beta}} \in \mathscr{H}^{\mathcal{C}} \mathscr{H}_{\omega_{1}, \omega} .
$$

So, it is natural to ask the following.

Question 1 When does it hold that $2^{\aleph_{\beta}} \in \mathscr{H}^{\mathcal{C}} \mathscr{H}_{\omega_{1}, \omega}$ ? In Part II we will concern ourselves with exactly this question. The importance of closure under the powerset operation is also stressed by the next theorem.

Theorem 4.10 If $\mathcal{C}$ is the smallest set of characterizable cardinals that contains $\aleph_{0}$ and is closed under successors, countable unions, countable products, and powerset, then it is also closed under powers.

Proof First observe that the following holds.

Claim $4 \quad$ If $\boldsymbol{\aleph}_{\alpha}^{\aleph_{\beta}} \in C$, then for all $\gamma<\omega_{1}, \boldsymbol{\aleph}_{\alpha+\gamma}^{\aleph_{\beta}} \in C$.

Proof This is proved as in the proof of Theorem 4.9 with the obvious modifications.

Recall from Definition 1.5 that $\boldsymbol{\aleph}_{\alpha}$ is the head of a cluster in $C$ if there are no $\beta, \gamma$ such that $\boldsymbol{\aleph}_{\beta} \in C, \gamma<\omega_{1}$, and $\boldsymbol{\aleph}_{\alpha}=\boldsymbol{\aleph}_{\beta+\gamma}$.

So, it suffices to prove that if $\boldsymbol{\aleph}_{\alpha}, \boldsymbol{\aleph}_{\beta} \in C$, then $\boldsymbol{\aleph}_{\alpha}^{\aleph_{\beta}} \in C$, for all $\boldsymbol{\aleph}_{\alpha}$-heads of clusters.

If $\boldsymbol{\aleph}_{\alpha} \leq \boldsymbol{\aleph}_{\beta}$, then $\boldsymbol{\aleph}_{\alpha}^{\boldsymbol{\aleph}_{\beta}}=2^{\boldsymbol{\aleph}_{\beta}} \in \mathcal{C}$. So, assume that $\boldsymbol{\aleph}_{\alpha}>\boldsymbol{\aleph}_{\beta} \geq \boldsymbol{\aleph}_{0}$, and proceed by induction on $\alpha$.

Since $\boldsymbol{\aleph}_{\alpha}$ is the head of a cluster and by the way that $C$ was defined, $\boldsymbol{\aleph}_{\alpha}$ is either a countable union, or a countable product of smaller cardinals, or the powerset of a smaller cardinal.

Case I. We have $\boldsymbol{\aleph}_{\alpha}=\sup _{n} \aleph_{\alpha_{n}}$.

Then

$$
\aleph_{\alpha}^{\aleph_{\beta}}=\left(\lim _{n} \boldsymbol{\aleph}_{\alpha_{n}}^{\aleph_{\beta}}\right)^{\omega}
$$

For every $n, \boldsymbol{\aleph}_{\alpha_{n}}^{\aleph_{\beta}} \in C$ by the inductive hypothesis, and the result follows by closure under countable products. 
Case II. We have $\boldsymbol{\aleph}_{\alpha}=\prod_{n} \boldsymbol{\aleph}_{\alpha_{n}}=\left(\sup _{n} \boldsymbol{\aleph}_{\alpha_{n}}\right)^{\boldsymbol{\aleph}_{0}}$.

If $\boldsymbol{\aleph}_{\alpha}=\sup _{n} \boldsymbol{\aleph}_{\alpha_{n}}$, then we fall under Case I. So, assume that $\boldsymbol{\aleph}_{\alpha}>\sup _{n} \boldsymbol{\aleph}_{\alpha_{n}}$. Then

$$
\boldsymbol{\aleph}_{\alpha}^{\aleph_{\beta}}=\left(\sup _{n} \boldsymbol{\aleph}_{\alpha_{n}}\right)^{\aleph_{\beta}}
$$

and the result follows.

Case III. We have $\boldsymbol{\aleph}_{\alpha}=2^{\boldsymbol{\aleph}_{\gamma}}$, some $\boldsymbol{\aleph}_{\gamma} \in C$.

Then $\boldsymbol{\aleph}_{\alpha}^{\boldsymbol{\aleph}_{\beta}}=2^{\boldsymbol{\aleph}_{\gamma} \cdot \boldsymbol{\aleph}_{\beta}}$.

Question 2 How much of the characterizable cardinals does $\mathcal{C}$ capture?

If we have $\mathrm{GCH}$, then obviously it captures everything. But are there any models

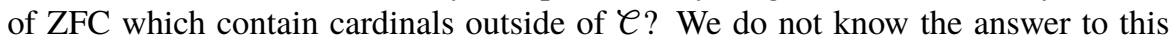
question. If the answer is negative, that is, $C=\mathcal{C} \mathscr{H}_{\omega_{1}, \omega}$, then we can tell all characterizable cardinals of a model quite easily. If the answer is positive, that is, $C \neq \smile \mathscr{H}_{\omega_{1}, \omega}$, then there exist cardinals outside of $\zeta$ and it would be interesting to find such examples.

The question whether $C=\mathcal{C} \mathscr{H}_{\omega_{1}, \omega}$ is also closely related to the following conjecture of Shelah.

Conjecture 2 (Shelah) If $\boldsymbol{\aleph}_{\omega_{1}}<2^{\aleph_{0}}$, then every $\mathscr{L}_{\omega_{1}, \omega}$-sentence with a model in power $\aleph_{\omega_{1}}$ has a model in power $2^{\aleph_{0}}$.

If the above conjecture is true and $\boldsymbol{\aleph}_{\omega_{1}}<2^{\aleph_{0}}$, then there is no characterizable cardinal $\kappa$ such that $\boldsymbol{\aleph}_{\omega_{1}} \leq \kappa<2^{\aleph_{0}}$.

\section{Some Counterexamples}

We provide some counterexamples to show that $\kappa \in \mathcal{C} \mathscr{H}_{\omega_{1}, \omega}$ does not imply cf $(\kappa) \in \mathcal{C} \mathscr{H}_{\omega_{1}, \omega}$, and $\kappa^{+} \in \mathcal{C} \mathscr{H}_{\omega_{1}, \omega}$ does not imply $\kappa \in \mathcal{C} \mathscr{H}_{\omega_{1}, \omega}$.

Theorem 5.1 $\mathcal{C} \mathscr{H}_{\omega_{1}, \omega}$ is not closed under predecessor or cofinality.

Proof We have to construct a counterexample. Shelah in [8] constructed a model where $2^{\aleph_{0}}>\boldsymbol{\aleph}_{\omega_{1}}$ and where no cardinal $\kappa$ with $\boldsymbol{\aleph}_{\omega_{1}} \leq \kappa<2^{\aleph_{0}}$ is characterizable. We say that in this case $\boldsymbol{\aleph}_{\omega_{1}}$ is the local Hanf number below $2^{\boldsymbol{\aleph}_{0}}$. This was done by adding $\boldsymbol{\aleph}_{\omega_{1}}$-many Cohen reals to a ground model that satisfies $\mathrm{GCH}$.

If we demand a little bit more here, we can take for instance that $2^{\boldsymbol{\aleph}_{0}}=\boldsymbol{\aleph}_{\omega_{1}+1}$. Then $\boldsymbol{\aleph}_{\omega_{1}+1}$ is characterizable, while $\boldsymbol{\aleph}_{\omega_{1}}$ is not. This proves the first part of the theorem.

Now, if we let $2^{\aleph_{0}}=\aleph_{\omega_{\omega_{1}+1}}$, then we get

$$
c f\left(2^{\aleph_{0}}\right)=c f\left(\aleph_{\omega_{\omega_{1}+1}}\right)=\boldsymbol{\aleph}_{\omega_{1}+1},
$$

which, again, is not a characterizable cardinal. This gives the second part of the theorem.

\section{Reformulations and Open Questions}

In this section we formulate a few open questions about $(\mathcal{M}, \mathcal{N})$-full structures in terms of functions.

Lemma 6.1 Let $\kappa, \lambda, \mu$ be cardinals with $\lambda \leq \kappa^{+}$, and let $\mathcal{M}$ be a model of size $\kappa$. The following are equivalent. 
(1) There exists an $(\mathcal{M}, \lambda)$-full structure $\mathcal{A}$ of size $\mu$.

(2) There exists a function $f: \mu^{2} \backslash\{(\alpha, \alpha) \mid \alpha<\mu\} \rightarrow \kappa$, and there exists a coloring function $c: \mu \rightarrow \lambda$ such that

(a) for every $\alpha, \beta<\mu, f(\alpha, \beta)=f(\beta, \alpha)$,

(b) for every $\alpha, \beta<\mu$, the set $T(\alpha, \beta)=\{\gamma<\mu \mid f(\alpha, \gamma)=f(\beta, \gamma)\}$ is finite,

(c) for every finite $X \subset \mu$, there exists some finite $Y \subset \mu$ which is closed under $T$; that is, for all $\alpha, \beta \in Y, T(\alpha, \beta) \in Y$,

(d) for every finite $Y, Z \subset \mu$ with $Y \subset Z$ and for every $g: Z^{2} \backslash\{(\alpha, \alpha) \mid$ $\alpha \in Z\}$ such that $g\left(y_{1}, y_{2}\right)=f\left(y_{1}, y_{2}\right)$, for all $y_{1} \neq y_{2} \in Y$, there exists a one-to-one mapping $i: Z \rightarrow \mu$ such that $\left.i\right|_{Y}=i d$ and for all $z_{1} \neq z_{2} \in Z, g\left(z_{1}, z_{2}\right)=f\left(i\left(z_{1}\right), i\left(z_{2}\right)\right)$, and

(e) (d) holds true even if $Z$ (and $Y$ ) is colored; that is, there exists a coloring function $d: Z \rightarrow \mu$ such that $d(y)=c(y)$ for all $y \in Y$, and we require now that $i$ has to satisfy the extra assumption for all $z \in Z$, $d(z)=c(i(z))$.

Proof $\quad{ }^{4}$ Let $\mathcal{A}$ be an $(\mathcal{M}, \lambda)$-full structure of size $\mu$. Since $|\mathcal{M}|=\kappa$, without loss of generality we can assume that the universe of $\mathcal{M}$ is actually $\kappa$, and we identify all $m \in \mathcal{M}$ with elements of $\kappa$. By Observation 3.7(c), $|\mathcal{A} \backslash(\mathcal{M} \cup \mathcal{N})|=\mu$, and we will identify all elements of $\mathcal{A} \backslash(\mathcal{M} \cup \mathcal{N})$ with elements of $\mu$. Let

$$
f(\alpha, \beta)=\gamma \quad \text { if and only if } \quad \mathcal{A} \models S(\gamma, \alpha, \beta)
$$

and

$$
c(\alpha)=\beta \quad \text { if and only if } \quad \mathcal{A} \models P(\beta, \alpha) .
$$

It is not hard to see that under this definition 2(b) follows from $\left(* 2^{\mathcal{M}, \mathcal{N}}\right), 2$ (c) is a reformulation of $(\mathcal{M}, \lambda)$-happiness, and 2(d) and 2(e) are reformulations of part (2) of the definition of $(\mathcal{M}, \lambda)$-fullness (see Definition 3.6).

For the inverse direction we work similarly.

\section{Notes}

(i) It follows from 2(d) that $f$ is onto $\kappa$. Otherwise, let $\alpha \in \kappa \backslash \operatorname{range}(f)$, and define a new finite function $g$ on some finite $Z$ such that $g\left(z_{1}, z_{2}\right)=\alpha$, for some $z_{1} \neq z_{2} \in Z$. By 2(d), there exists some $i: Z \rightarrow \mu$ such that $\alpha=g\left(z_{1}, z_{2}\right)=f\left(i\left(z_{1}\right), i\left(z_{2}\right)\right)$, a contradiction.

(ii) In particular, $f$ being onto $\kappa$ implies that $\kappa \leq \mu$.

(iii) Working similarly to (i) and using 2(e), we can prove that the coloring function $c$ is onto $\lambda$. In particular, $\lambda \leq \mu$. The details are left to the reader.

Definition 6.2 A function that satisfies 2(a)-2(e) of Lemma 6.1 will be called a $(\kappa, \lambda, \mu)$-full function.

If $f$ is a $(\kappa, \lambda, \mu)$-full function, then by Lemma 3.9 we infer that $\mu \leq \kappa^{+}$. Combining with the fact that $\kappa \leq \mu$ we remain with only two possibilities: either $\mu=\kappa$ or $\mu=\kappa^{+}$.

Open Question 1 Given an infinite cardinal $\kappa$ and $\lambda \leq \kappa^{+}$, find the maximum $\mu$ such that there exists a $(\kappa, \lambda, \mu)$-full function.

As we mentioned this maximum $\mu$ is either $\kappa$ or $\kappa^{+}$, and we have examples for either case. If $\kappa^{\omega}=\kappa$, then by Lemma 3.20, there are no $\left(\kappa, \lambda, \kappa^{+}\right)$-full functions. In particular, the maximum $\mu$ equals $\kappa$. If $\kappa=\aleph_{0}$, then it follows from [3, 
Corollary 3.2] that there exists an $\left(\boldsymbol{\aleph}_{0}, \boldsymbol{\aleph}_{0}, \boldsymbol{\aleph}_{1}\right)$-full function, which implies that the maximum $\mu$ is $\aleph_{1}$ in this case.

Open Question 2 Does the existence of a $\left(\kappa, 1, \kappa^{+}\right)$-full function imply the existence of a $\left(\kappa, \lambda, \kappa^{+}\right)$-full function for some infinite $\lambda \leq \kappa^{+}$? The cases $\lambda=\kappa$ and $\lambda=\kappa^{+}$are the most interesting ones.

\section{Notes}

1. In the last section, this question appears reformulated as Open Question 2.

2. $\mathcal{A}$ here actually refers to $(\mathcal{A} \backslash\{1\}) \cup \kappa^{+}$, but as in part (a), we will just write $\mathcal{A}$ for convenience.

3. The idea of this proof was communicated to the author by professor Magidor.

4. The way we define $f$ in this proof is similar to the way we defined the family of functions $\mathcal{F}$ in the proof of Lemma 3.20.

5. Professor Hjorth died unexpectedly in January 2011.

\section{References}

[1] Baumgartner, J. E., "The Hanf number for complete $L_{\omega_{1}, \omega}$ sentences (without GCH)," Journal of Symbolic Logic, vol. 39 (1974), pp. 575-78. MR 0363796. 534, 535, 542

[2] Hanf, W., "Incompactness in languages with infinitely long expressions," Fundamenta Mathematicae, vol. 53 (1963/1964), pp. 309-24. MR 0160732. 534

[3] Hjorth, G., "Knight's model, its automorphism group, and characterizing the uncountable cardinals," Journal of Mathematical Logic, vol. 2 (2002), pp. 113-44. Zbl 1010.03036. MR 1900550. DOI 10.1142/S0219061302000084. 535, 536, 537, 538, 549

[4] Keisler, H. J., Model Theory for Infinitary Logic: Logic with Countable Conjunctions and Finite Quantifiers, vol. 62 of Studies in Logic and the Foundations of Mathematics, North-Holland, Amsterdam, 1971. MR 0344115. 533

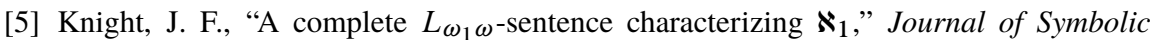
Logic, vol. 42 (1977), pp. 59-62. MR 0491141. 535

[6] Malitz, J., "The Hanf number for complete $L_{\omega_{1}, \omega}$ sentences," pp. 166-81 in The Syntax and Semantics of Infinitary Languages (Los Angeles, 1967), vol. 72 of Lecture Notes in Mathematics, Springer, New York, 1968. 534, 535, 536, 542

[7] Scott, D., "Logic with denumerably long formulas and finite strings of quantifiers," pp. 329-41 in Theory of Models (Berkeley, 1963), North-Holland, Amsterdam, 1965. MR 0200133. 533

[8] Shelah, S., "Borel sets with large squares," Fundamenta Mathematicae, vol. 159 (1999), pp. 1-50. Zbl 0941.03052. MR 1669643. 548

[9] Souldatos, I., "Linear orderings and powers of characterizable cardinals," Annals of Pure and Applied Logic, vol. 163 (2012), pp. 225-37. Zbl 1250.03060. MR 2871267. DOI 10.1016/j.apal.2011.09.002. 533 


\section{Acknowledgments}

I would like to thank the Department of Mathematics and Statistics of the University of Melbourne, Australia, for their kind hospitality for the whole academic year 2006-2007. This paper was written while visiting professor Greg Hjorth, ${ }^{5}$ my thesis advisor at the above university.

Mathematics Department

University of Detroit Mercy

4001 W. McNichols

Detroit, Michigan 48221

USA

souldaio@udmercy.edu 